\title{
Nonlinear optical switching matrix
}

\author{
Gang Jun Liu, Jun Liu, Bin Ming Liang, Qu Li, and Guo Liang Jin \\ Department of Physics, Shanghai Jiao Tong University, Dongchuan Road 800, Minhang, Shanghai 200240, China
}

Received January 22, 2003

\begin{abstract}
We show that nonlinear directional couplers with variable coupling coefficients can be used to compose a special kind of switching matrix. The switching matrix can be controlled by the signal itself or by a strong pump. Light routing is realized by adjustment of the power of the signal or the pump. Because light routing is based on the optical Kerr effect, ultrafast routing is possible. (C) 2003 Optical Society of America

OCIS codes: $190.4360,230.1150,130.3120$.
\end{abstract}

The potential applications of nonlinear directional couplers (NLDCs) in all-optical signal processing have attracted a great deal of research interest since they were first introduced by Jensen in 1982. ${ }^{1}$ Several variations of the NLDC, such as $\mathrm{Y}$ junctions, ${ }^{2} \mathrm{X}$ junctions, ${ }^{3}$ and bent couplers, ${ }^{4-6}$ have been proposed since then. Usually, these devices can be used as, e.g., optical switches, optical limiters, optical intensity discriminators, and logic gates. When they are used as switching devices, NLDCs can be self-switching devices or pump-controlled switches. For self-switching devices, the signal power should be changed before the signal enters the device, and for pump-controlled switches, pump light at a different wavelength or with a different polarization will enter the device, with prescribed power, overlapping the signal.

The dramatic success of optical technology in telecommunication systems encouraged researchers to develop technologies for optical switching systems. Optical switching matrices, as key components in the optical communication systems, are the signal routing modules in the optical layer. There are now several types of optical switching matrix that are based on different mechanisms, such as microelectromechanical systems-based optical switching, bubble switching, thermo-optical switching, and electro-optic switching matrices. ${ }^{7,8}$ These matrices have different switching times ranging from microseconds to nanoseconds. Switches based on the optical Kerr effect could yield switching as fast as a picosecond. However, there has not yet been any research on realization of a switching matrix based on the Kerr effect, to the best of our knowledge. In this Letter we propose a kind of switching matrix based on the optical Kerr effect. It is not just a simple combination of identical switch units as in the conventional switching matrix. In this special matrix the switch units are not identical, and signal routing is realized by adjustment of the signal power (self-switching) or the power of the pump (pump-controlled switching).

The switching characteristics of the $\mathrm{X}$ junction and the bent coupler are very interesting. For multiplecoupling-length devices, both kinds of coupler show multiple digital switching characteristics at high input powers. ${ }^{3,9}$ Generally, the multiple switching characteristics of these devices are unfavorable for switching because of pulse breakup. ${ }^{10}$ However, the use of solitonlike pulses ${ }^{11}$ or of control pulses with different wavelength and different pulse durations ${ }^{12}$ could suppress pulse breakup. So the multiple switching characteristics of the NLDC could allow it to be used as an optical switching device. We show below that the multiple-coupling-length Gaussian-type coupling coefficient NLDC, or equivalently, the multiplecoupling-length bent coupler, has special switching characteristics that one can exploit to create a special kind of switching matrix.

Figure 1 shows a schematic diagram of a $4 \times 4$ switching matrix composed of four nonlinear couplers. Couplers 1 and 2 are identical, and couplers 3 and 4 are different from couplers 1 and 2 and from each other. Suppose that the straight-through transmission curves of the four couplers have the characteristics shown in Fig. 2. The switching is controlled by the signal power (or the pump power) at the input port. First, we analyze the situation in which the input power of the signal (or the power of the pump) is in the range $\mathrm{P} 0-\mathrm{P} 1$. In this power range couplers 1 (2) and 3 are in the bar state, and coupler 4 is in the cross state. If the light is input from port $\mathrm{A}$, the operating switching units are couplers 1 and 3, which are both in the bar state, and the light will be output from A'. Similarly, light of power P0-P1 that is input from $\mathrm{B}$ will be output from $\mathrm{D}^{\prime}$, input from $\mathrm{C}$ will be output from $\mathrm{B}^{\prime}$, and input from $\mathrm{D}$ will be output from $\mathrm{C}^{\prime}$. Accordingly, when the power is in the range $\mathrm{P} 1-\mathrm{P} 2$, couplers 1 (2) and 4 are in the bar state and coupler 3 is in the cross state. When input power is in the range $\mathrm{P} 2-\mathrm{P} 3$, the couplers 1 (2) and 3 are in the cross state and coupler 4 is in the bar state. When input power is in the range P3-P4, the couplers 1 (2) and 4 are both in the cross state and coupler 3 is in the bar state. We summarize the routing of the matrix in Table 1 . The power could be the signal power or the control pump power. From the table, it can be clearly seen that we can route the

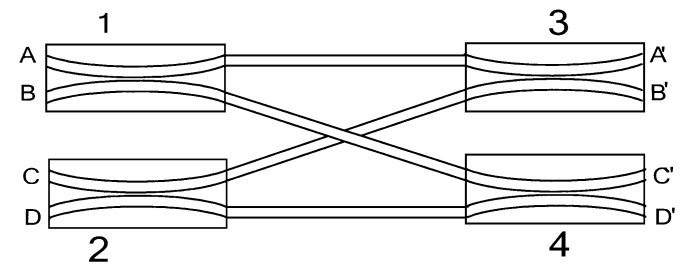

Fig. 1. Schematic of the nonlinear switching matrix. 


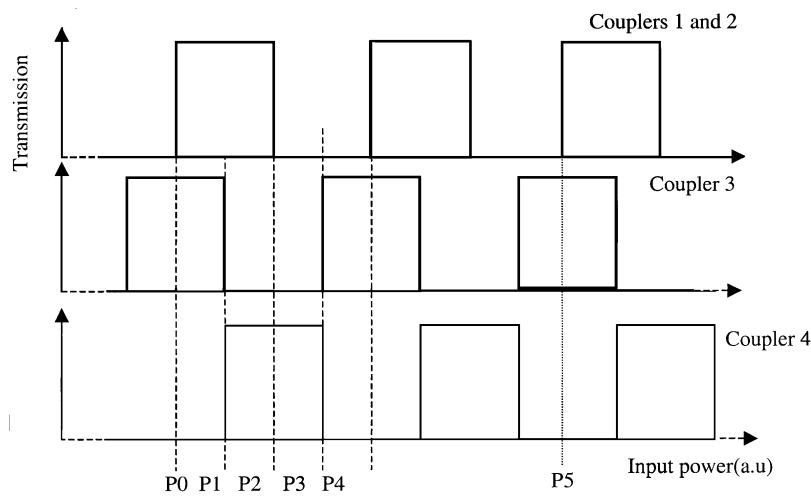

Fig. 2. Ideal straight-through transmission of the switching units in the matrix.

Table 1. Routing Table of the Switching Matrix

\begin{tabular}{ccccc} 
& \multicolumn{4}{c}{ Input-Power Range } \\
\cline { 2 - 5 } Input Port & $\mathrm{P}$ - 1 1 & $\mathrm{P} 1-\mathrm{P} 2$ & $\mathrm{P} 2-\mathrm{P} 3$ & $\mathrm{P} 3-\mathrm{P} 4$ \\
\hline $\mathrm{A}$ & $\mathrm{A}^{\prime}$ & $\mathrm{B}^{\prime}$ & $\mathrm{C}^{\prime}$ & $\mathrm{D}^{\prime}$ \\
$\mathrm{B}$ & $\mathrm{D}^{\prime}$ & $\mathrm{C}^{\prime}$ & $\mathrm{B}^{\prime}$ & $\mathrm{A}^{\prime}$ \\
$\mathrm{C}$ & $\mathrm{B}^{\prime}$ & $\mathrm{A}^{\prime}$ & $\mathrm{D}^{\prime}$ & $\mathrm{C}^{\prime}$ \\
$\mathrm{D}$ & $\mathrm{C}^{\prime}$ & $\mathrm{D}^{\prime}$ & $\mathrm{A}^{\prime}$ & $\mathrm{B}^{\prime}$ \\
\hline
\end{tabular}

signal from any one of the four input ports (A-D in Fig. 1) to any one of the four output ports $\left(A^{\prime}-D^{\prime}\right.$ in Fig. 1) by properly choosing the input power.

Below, we show how to realize the transmission curves of Fig. 2 by use of nonlinear bent couplers. The nonlinear bent coupler is a coupler with a Gaussiantype varying coupling coefficient. Its operation can be described by the following coupled equations ${ }^{9}$ :

$$
\begin{aligned}
& \frac{\partial c_{1}}{\partial z}=i \kappa_{0} \exp \left(-\frac{z^{2}}{b^{2}}\right) c_{2}+i \gamma\left|c_{1}\right|^{2} c_{1}, \\
& \frac{\partial c_{2}}{\partial z}=i \kappa_{0} \exp \left(-\frac{z^{2}}{b^{2}}\right) c_{1}+i \gamma\left|c_{2}\right|^{2} c_{2},
\end{aligned}
$$

where $c_{1}$ and $c_{2}$ are the normalized complex amplitudes of the mode fields of waveguides 1 and 2 , respectively; i.e., $\left|c_{1}\right|^{2}=P_{1},\left|c_{2}\right|^{2}=P_{2}$, where $P_{1}$ and $P_{2}$ are the power in waveguides 1 and 2 , respectively. $\kappa_{0} \exp \left(-z^{2} / b^{2}\right)$ represents the linear coupling between the two waveguides, where $\kappa_{0}$ is the maximum coupling coefficient corresponding to the center of the coupler, $b$ is the distance at which the coupling coefficient decreases to $1 / e$ of the maximum value, and the length of the device is chosen to be $6 b$, so that the decoupling condition can be satisfied at the input and output ports. $\gamma=k_{0} n_{2} / A_{\text {eff }}$, where $n_{2}$ is the nonlinear index coefficient and $A_{\text {eff }}$ is the effective cross-section area of the waveguide mode. The last terms on the right-hand sides of Eqs. (1a) and (1b) arise from self-phase modulation. Cross-phase modulation is neglected.

For nonlinear bent couplers, the power is almost the same for consecutive switching, the cutoff switching power is proportional to the maximum coupling coefficient of the coupler, and the switching curve is very square at relatively high powers for multiple- coupling-length couplers. ${ }^{9}$ Based on the special characteristics of the nonlinear bent coupler, we can realize the transmission curves shown in Fig. 2 by properly choosing the coupler parameters. Figure 3(a) shows the straight-through transmission curves of three nonlinear bent couplers. The dotted curve is the straight-through transmission curve of a 20-coupling-length nonlinear bent coupler with maximum coupling coefficient $\kappa_{0}$. The input power is normalized to $P_{c}=4 \kappa_{0} / \gamma$. The solid curve corresponds to a 19.5-coupling-length nonlinear bent coupler with maximum coupling coefficient $0.975 \kappa_{0}$. The dashed curve is for a 20.5-coupling-length nonlinear bent coupler with maximum coupling coefficient $1.025 \kappa_{0}$. These parameters are chosen such that the power difference between two consecutive switchings is almost the same for the three couplers and the three curves are staggered. Figure 3(b) shows the higher-input-power part of Fig. 3(a). Comparing Fig. 3(b) with Fig. 2, we find that the solid curve in Fig. 3(b) corresponds to the transmission curve of coupler 3 in Fig. 2, the dotted curve corresponds to

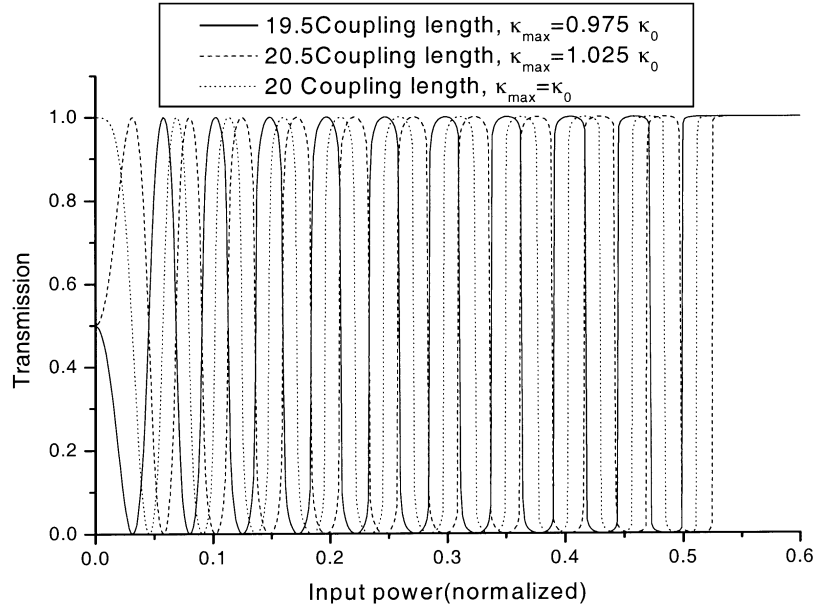

(a)

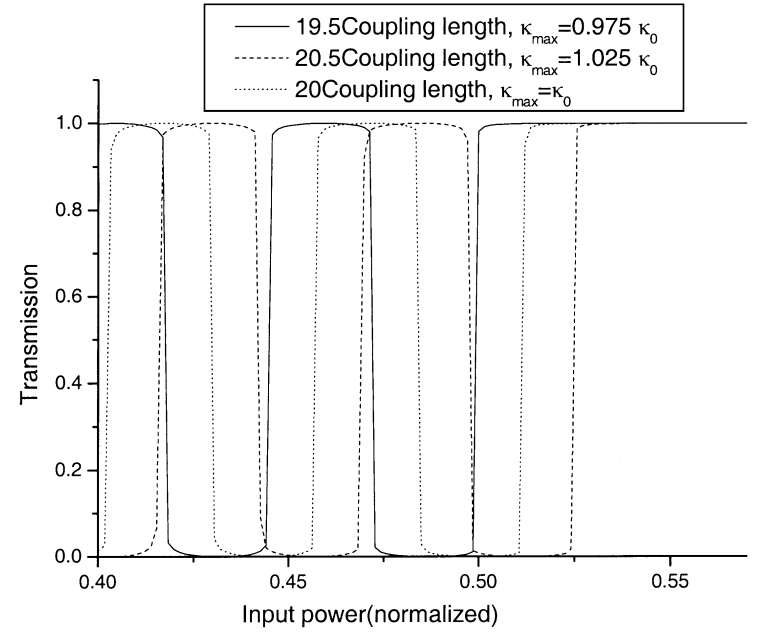

(b)

Fig. 3. Transmission curves of three Gaussian-type variable-coupling-coefficient NLDCs: (a) total transmission curves, (b) transmission curves of the high-inputpower part. 
the transmission curve of couplers 1 and 2 , and the dashed curve corresponds to that of coupler 4 . From Fig. 3(b), one can see that the transmission curves of the nonlinear bent couplers are very close to the ideal curves shown in Fig. 2. It also can be seen that more than one power range can be adopted to control the matrix. The above analysis of the routing of the matrix is based on the self-switching condition. Our simulation shows that, for the pump-controlled switching condition, the signal has almost the same transmission curve as that of the pump as long as they overlap at the input port. So our results are also suitable for the pump-controlled switching condition.

For a 1-coupling-length conventional NLDC, the switching power approximately equals the critical power, $P_{c}=4 \kappa_{0} / \gamma^{1}{ }^{1}$ One can see from Fig. 3 that the power needed to control the matrix is smaller than that of the 1-coupling-length conventional NLDC with the same values $\kappa_{0}$ and $\gamma$. However, this power is achieved at the cost of a longer device. If we adopt the same parameters as in Ref. 3, the index of the waveguide is 1.55 , the index of the substrate is 1.5 , the width of the waveguide is $1.5 \mu \mathrm{m}$, the wavelength is $1.3 \mu \mathrm{m}$, and $n_{2}=7.5 \times 10^{-14} \mathrm{~m}^{2} / \mathrm{W}$, for a 20 coupling-length bent coupler with $\kappa_{0}=20,000 \mathrm{~m}^{-1}$, the minimum length of a single coupler is $\sim 5.32 \mathrm{~mm}$, the radius of the bent arc is $\sim 1.15 \mathrm{~m}$, and the power needed to control the matrix is $\sim 1.1 \mathrm{~W}$ for $A_{\text {eff }}=10 \mu \mathrm{m}^{2}$. From Fig. 3(b), it can be seen that the cross talk of the matrix is dependent on the power of the signal (or the power of the pump). The loss has a great effect on the device performance. Loss will decrease the power, and if the power is not in the proper power range, correct routing cannot be realized. On the other hand, loss and index saturation will deteriorate the switching curve. ${ }^{6}$ The switching curve will not be so square as shown in Fig. 3, and the power difference between two consecutive switchings will not be identical. So both the loss and the index saturation will increase cross talk and even disable switching. In real applications, proper material should be chosen so that the loss is low and the saturated index is high enough.

The switching matrix proposed in this Letter is based on a novel routing scheme. It is in fact different from a conventional switching matrix in several respects. In a conventional switching matrix, it is necessary to control different matrix units to route the signal. However, in this nonlinear switching matrix, the power of the signal itself or the power of the control pump determines the routing of the signal. The nonlinear switching matrix is based on the optical Kerr effect, so the switching could be as fast as a picosecond. The matrix discussed above is in fact a $4 \times 4$ banyan network, so we could scale it to construct an $8 \times 8$ banyan network as in conventional banyan networks. The first stage of the $8 \times 8$ banyan network can be four identical bent couplers whose power difference between consecutive switchings is twice that of the two $4 \times 4$ matrices in the second stage. So the four identical couplers in the first stage of the $8 \times 8$ banyan network are all in the bar (cross) state in the power range $\mathrm{P} 0-\mathrm{P} 4$ and are all in the cross (bar) state in the power range $\mathrm{P} 4-\mathrm{P} 5$ of Fig. 2. These four identical couplers can also be realized with nonlinear bent couplers. Accordingly, we can construct an $N \times N$ matrix from $N / 2$ identical couplers and two $N / 2 \times N / 2$ matrices.

In conclusion, we have shown that nonlinear directional couplers, which have been used as switches, can be used to construct a novel kind of switching matrix. This matrix is based on the special switching characteristics of the multiple-coupling-length Gaussian-type variable-coupling-coefficient NLDC. We have analyzed the principle of a $4 \times 4$ switching matrix and proposed a way to realize it. The cross talk and scalability of the matrix are discussed.

\section{G. J. Liu's e-mail address is lgj@sjtu.edu.}

\section{References}

1. S. M. Jensen, IEEE J. Quantum Electron. QE-18, 1580 (1982).

2. Y. Silberberg and B. G. Sfez, Opt. Lett. 13, 1132 (1988).

3. J. P. Sabini, N. Finlayson, and G. I. Stegeman, Appl. Phys. Lett. 55, 1176 (1989).

4. V. Leutheuser, U. Langbein, and F. Lederer, Opt. Commun. 75, 251 (1990).

5. H. Hatami-Hanza and P. L. Chu, Opt. Commun. 119, 347 (1995).

6. G. J. Liu, B. M. Liang, G. L. Jin, and Q. Li, Appl. Opt. 41, 5022 (2002).

7. N. Keil, H. H. Yao, C. Zawadzki, and B. Strebel, Electron. Lett. 31, 403 (1995).

8. H. Okayama and M. Kawahara, Electron. Lett. 30, 1128 (1994).

9. G. J. Liu, B. M. Liang, Q. Li, and G. L. Jin, Opt. Commun. 218, 113 (2003).

10. G. I. Stegeman and E. M. Wright, Opt. Quantum Electron. 22, 95 (1990).

11. M. Romagnoli, S. Trillo, and S. Wabnitz, Opt. Quantum Electron. 24, S1237 (1992).

12. J. S. Aitchison, A. Villeneuve, J. U. Kang, and G. I. Stegeman, in Towards Terabit Transmission, IEE Seminar Digest 110 (Institution of Electrical Engineers, London, 1995), pp. 7/1-7/6. 